\title{
Self-sustained current oscillations in spin-blockaded quantum dots
}

\author{
B. Hu (胡滨) ${ }^{1}$ and X. R. Wang (王向荣) $)^{1,2, *}$ \\ ${ }^{1}$ Physics Department, The Hong Kong University of Science and Technology, Clear Water Bay, Kowloon, Hong Kong \\ ${ }^{2}$ School of Physics, Wuhan University, Wuhan, P. R. China
}

(Received 18 July 2012; published 23 January 2013)

\begin{abstract}
Self-sustained current oscillations observed in spin-blockaded double quantum dots are explained as a consequence of periodic motion of dynamical nuclear spin polarization (along a limit cycle) under an external magnetic field and a spin-transfer torque. Based on the Landau-Lifshitz-Gilbert equation, it is shown that a sequence of semistable limit cycle, Hopf, and homoclinic bifurcations occur as the external field is tuned. The divergent period near the homoclinic bifurcation explains well why the period in experiments can be many orders of magnitude longer than all microscopic time scales.
\end{abstract}

DOI: 10.1103/PhysRevB.87.035311

PACS number(s): 73.63.Kv, 72.25.Rb, 76.60.-k, 05.45.-a

\section{INTRODUCTION}

Quantum dots, also known as artificial atoms, have many properties of natural atoms such as discrete energy levels and shell structures. ${ }^{1,2}$ The physics involved in quantum dots is very rich because of tunability and comparability of three energy scales: level spacing, Coulomb interaction, and thermal energy. Unlike natural atoms, quantum dots allow transport measurements. Many interesting transport phenomena such as resonant tunneling, Coulomb blockade, spin blockade, Kondo effects, quantum conductance, etc., have been observed and explained. Applications in nanoelectronics, spintronics, and quantum computing due to possible long coherence time have been proposed and in some cases implemented. It is known that both the nuclear and electron spin degrees of freedom in semiconductor nanostructures can be manipulated by the hyperfine interactions. ${ }^{3-6}$ In the endeavor of using hyperfine interaction to manipulate the electron transport in quantum dots, one long-term unexplained intriguing phenomenon was observed by Ono and Tarucha in 2004 (Ref. 6) in spinblockaded double quantum dots: tunneling current $I$ under a dc bias, as artistically shown in Fig. 1, oscillates with a period up to minutes under certain conditions. In this paper, we show that dynamical nuclear spin polarization (DNSP), governed by the generalized Landau-Lifshitz-Gilbert equation, can oscillate with time under a dc bias in a magnetic field window. The back-effect of the DNSP oscillation on the electron tunneling leads to the experimentally observed self-sustained current oscillation.

A coherent theory should be consistent with the following experimental findings: (1) Oscillation was observed only in the spin-blockaded regime in a magnetic field window accompany by a current jump. (2) Both the period and amplitude increase with the field before the oscillation disappears. (3) The oscillation period varies from seconds (apparatus $\operatorname{limit}^{6}$ ) to several minutes, many orders of magnitude longer than the typical spin precession time in a Tesla field and electron tunneling time through the double quantum dots. ${ }^{6}$ (4) The oscillation is closely related to the motion of the DNSP. ${ }^{6-10}$ So far, no existing theoretical attempts ${ }^{7-11}$ can satisfactorily explain all these features. It is clear that the oscillation theory for semiconductor superlattices ${ }^{12}$ is not applicable because the period would be on the order of $100 \mathrm{~ns}$ electron tunneling time, instead of observed seconds and minutes. Thermal and impurity effects were also ruled out. ${ }^{6}$ Although the nuclear spins in a quantum dot are known to have a very long (up to seconds) relaxation time, the relaxation process is not a periodic motion so that it can not be the cause of the oscillation.

\section{MODEL AND METHOD}

In order to construct a sensible model, let us examine the plausible microscopic process of electrons and nuclei in spin-blockaded double quantum dots. In the experiment, ${ }^{6}$ two vertical disklike quantum dots (InGaAs-AlGaAs multilayer structure) are weakly connected in series between source and drain [illustrated in Fig. 1(a)]. Four possible configurations in the spin-blockaded regime are shown in Fig. 1(b): One electron is trapped in the right dot (configuration A). The second electron, hopping from the source lead to the left dot, forms either spin singlet (B) or triplet (D) states. Electron tunneling cycle $\mathrm{A} \rightarrow \mathrm{B} \rightarrow \mathrm{C} \rightarrow \mathrm{A}$ is allowed, while tunneling is blockaded in $\mathrm{D}$, and here $\mathrm{C}$ is a spin-singlet state of two electrons on the right dot. The three triplet states $\left(\left|T^{0}\right\rangle,\left|T^{ \pm}\right\rangle\right)$ are degenerated in the absence of an external magnetic field. As shown in Fig. 1(c), in the presence of interdot tunneling at finite external field, the spin triplets are below the singlet state $|S\rangle$ by an energy $J$ depending on source-drain bias. ${ }^{7,13,14,20}$ Increasing the external field will lift the triplet degeneracy, and $|S\rangle$ and $\left|T^{-}\right\rangle$states anticross at a field $B_{r}$ around $0.5 \mathrm{~T}^{6}$ Due to the spin blockade and weak coupling of dots with two leads, a leakage current of order of $1 \mathrm{pA}$, corresponding to 100 ns electron tunneling time, exists. The current experiences a jump near $B_{r}$ because spin blockade is partially removed by spin flipping and the transition from configuration $\mathrm{D}$ to $\mathrm{B}$ is possible. The longitudinal component of this spin-flip process dynamically polarizes the nuclear spins, resulting in DNSP with a magnetization $\vec{M} \cdot{ }^{13}$ This spin-flip process mediates also an effective transversal spin transfer from electron spins to the nuclear spins which can affect DNSP dynamics. Adopting the view that dynamical motion of DNSP is responsible to the observed self-sustained current oscillation, we concentrate on the DNSP dynamics under the influence of both magnetic field and the above spin-flip process mediated spin-transfer torque.

It is well known that the Landau-Lifshitz-Gilbert equation governs the generic dynamics of a macrospin preserving its magnitude while the so-called Landau-Lifshitz-Bloch 
(a)

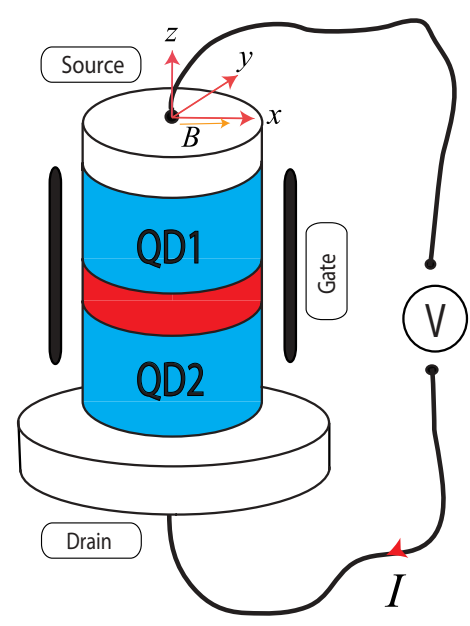

(b)
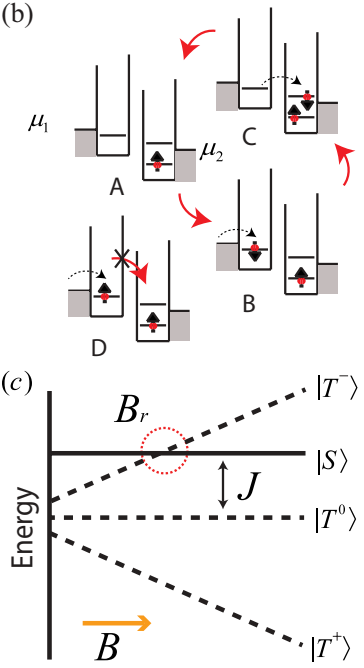

FIG. 1. (Color online) (a) Schematic setup of spin-blockaded double quantum dots device. Current $I$ pass through dots, QD1 and QD2 in series, under a dc bias $V$. External field is applied along the $x$ direction. (b) Four possible configurations in spin-blockaded regime. One spin-up electron is in the right quantum dot below the chemical potentials $\left(\mu_{i}, i=1,2\right)$ in configuration $\mathrm{A}$. In configuration $\mathrm{B}$, two electrons each on one of the two dots form a spin-singlet state. Configuration $\mathrm{C}$ is a spin-singlet state of two electrons in the right dot. Spin-blockaded configuration $\mathrm{D}$ is a spin-triplet state of two dots with one electron on each. (c) Schematic of field dependence of triplet and singlet states. Triplet state $\left|T^{-}\right\rangle$cross with singlet state $|S\rangle$ at field $B_{r} \approx 0.5 \mathrm{~T}$ (red circle). It is noted that the triplets are below the singlet at finite magnetic field in the spin-blockade region (Refs. 7, 13, and 20).

equation is for the dynamics of a macrospin whose magnitude can also vary. For simplicity and the well-known fact that the Landau-Lifshitz-Gilbert equation with the Slonczewski torque has oscillatory solution, we assume the following dynamics for $\vec{m}=\vec{M} /|\vec{M}|($ Ref. 15):

$$
\frac{d \vec{m}}{d t}=-\vec{m} \times \vec{H}_{\mathrm{eff}}+\alpha \vec{m} \times \frac{d \vec{m}}{d t}+a \vec{m} \times(\vec{m} \times \hat{x}) .
$$

Here, $t$ is in the units of $(\gamma M)^{-1}$, order of submicron second for GaAs with $M=10^{6} \mathrm{~A} / \mathrm{m}$ and the nuclear gyromagnetic ratio $\gamma=10(\mathrm{~A} \mathrm{~s} / \mathrm{m})^{-1} \cdot{ }^{16}$ The first term on the right-hand side of Eq. (1) describes the Larmor precession around the effective field $\vec{H}_{\text {eff }}=\vec{H}-D \vec{m}$ from both external magnetic field $\vec{H}$ along the $x$ axis, and demagnetization field $D \vec{m}$ (in the units of $|M|$ ). The presence of this term, depending on the DNSP distribution in the dots, is due to the magnetic charges on the sample boundary. Under uniform DNSP distribution approximation, the cylindrical disklike dots in experiment ${ }^{6}$ have diagonal demagnetization factors with $D_{x} \approx D_{y} \ll D_{z}$. In this paper, we assume $D_{x}=D_{y}=0, D_{z}=1$. In reality, a small difference between $D_{x}$ and $D_{y}$ may exist either due to the inhomogeneity or deviation of dots from the perfect cylindrical shape. Our numerical results show that the physics reported here remain the same for $D_{x} \neq D_{y}$. The second term in the right-hand side of Eq. (1) is the phenomenological Gilbert damping with a dimensionless constant $\alpha$. This empirical term, universally presented in all systems coupled to other outside degrees of freedom and testified by various experiments, has multiorigins, including eddy current in a bulky magnetic metal, the spin-lattice interaction, and spin-orbital coupling. Unlike the dipole-dipole relaxation which is longitudinal, the Gilbert damping preserves spin magnitude. The third term is the Slonczewski torque (per spin) which is universal in spin exchange between two spin baths with different polarizations. ${ }^{17}$ In the current setup, it is originated from the biased electron-nuclear spin flip-flop due to transition from $\left|T^{-}\right\rangle$to $|S\rangle$ mentioned earlier [Fig. 1(c)]. The Slonczewski coefficient $a=\eta W / N$ is proportional to transition rate $W$ from $\left|T^{-}\right\rangle$to $|S\rangle$ and inversely proportional to the polarized nuclear number $N \sim 10^{5}-10^{6}$. Dimensionless coefficient $\eta=S_{\perp} / \hbar$ measures the average spin angular moment quanta transferred from one electron to nuclei. In the Fermi golden rule approximation, $W$ reads as $^{8,11,18}$

$$
W=W_{0} \frac{\Gamma^{2}}{\Delta E^{2}+\Gamma^{2}} \times \xi
$$

where

$$
\xi= \begin{cases}1, & \Delta E>0 \\ \exp \left(\frac{\Delta E}{k_{B} T}\right), & \Delta E<0\end{cases}
$$

$\Delta E \equiv\left|g \mu_{B} B\right|-J$ is the level spacing between $\left|T^{-}\right\rangle$and $|S\rangle$ states. Note that the back-effect of DNSP on $\Delta E$ is neglected here. The inclusion of this effect is expected to make some quantitative modifications on the results reported below, but shall not change any physics. The effective Lande $g$ factor for GaAs is $g=-0.44 .{ }^{19} k_{B}$ is the Boltzmann constant and $T=$ $1.8 \mathrm{~K}$ is the experimental temperature. The level broadening $\Gamma$ of state $\left|T^{-}\right\rangle$is order of phonon energy of $\mu \mathrm{eV},{ }^{11}$ and $W_{0} \sim$ $10^{3}-10^{4} \mathrm{~s}^{-1}$ (Ref. 20) is the typical resonant spin flip-flop rate.

\section{RESULTS AND DISCUSSIONS}

In the absence of the external field, all points on the equator of the $\vec{m}$ sphere are marginal stable fixed points while $\vec{m}=(0,0, \pm 1)$ are unstable. Under a very small field, $\vec{m}=(-1,0,0)$ becomes the only saddle point (SP in Fig. 2$)$ and $\vec{m}=(1,0,0)$ the only stable attractor $\left(P_{+}\right.$in Fig. 2$)$. The two unstable fixed points (one of them is $P_{-}$in Fig. 2) move toward SP along the big cycle in the $x-z$ plane as B increases [indicated by the arrow in Fig. 2(a)]. In terms of energy, the $\alpha$ term is always an energy sinker while the $a$ term could be both energy sinker and source. ${ }^{15}$ In the vicinity of $P_{+}$, the $a$ term serves as an energy source that can be seen from the fact that the $a$ term tends to push the system away from $P_{+}$. As $B$ approaches $B_{r}$, the $a$ term may become large enough to destabilize $P_{+}$. This is confirmed by the standard stability analysis ${ }^{21}$ by calculating the Liapunov exponent at $P_{+}$. The Liapunov exponents at $P_{+}$ become positive at about $B=0.42 \mathrm{~T}$, and the system has no stable fixed point at this value. This entails the existence of limit cycle(s) in two dimensions (current case). Indeed, our numerical calculations support this scenario. Figure 2 shows the locations of various attractors of Eq. (1) at various $B$. For a field much smaller than $B_{r}$ [Fig. 2(a)], all phase flows end at $P_{+}$, the only stable fixed point of the system. At a critical field $B_{0}$, slightly smaller than $0.40 \mathrm{~T}$, the system undergoes a semistable limit-cycle bifurcation [shown in Fig. 2(b) at 


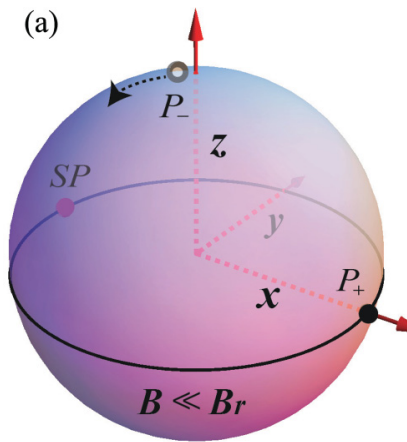

(b)
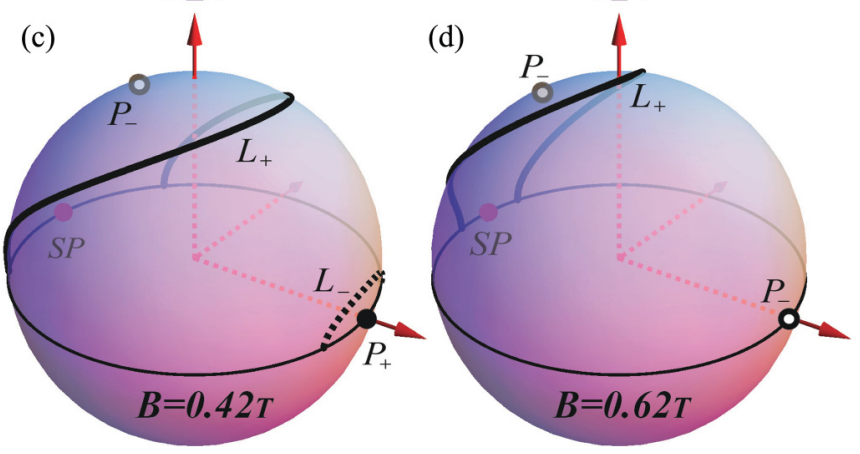

FIG. 2. (Color online) Change of attractors as external magnetic field increases [from (a) to (b)]: Only fix points/limit cycles on the upper sphere were showed for clarity. Solid (dashed) black line $L_{+}$ $\left(L_{-}\right)$denotes stable (unstable) limit cycle. $P_{+}\left(P_{-}\right)$labels stable (unstable) fixed point, and red dot SP is the saddle point at $\vec{m}=$ $(-1,0,0)$. (a) For $B \ll B_{r}$, the only stable attractor is $P_{+}$; (b) $B=$ $0.40 \mathrm{~T}$, slightly above the critical field at which semistable limit-cycle bifurcation occurs and $L_{+}$and $L_{-}$are generated; (c) $B=0.42 \mathrm{~T}$, slightly below subcritical Hopf bifurcation field at which $L_{-}$and $P_{+}$ merges and becomes an unstable fixed point; (d) $B=0.62 \mathrm{~T}$, slightly below the homoclinic bifurcation field at which $L_{+}$touches SP.

which a pair of limit cycles, one stable (solid black line) and the other unstable (dashed line), appear simultaneously. The two limit cycles move in opposite direction as the field increases further. At a critical value $B=B_{1}$ around $0.42 \mathrm{~T}$, the unstable cycle merges with $P_{+}$, undergoing a subcritical Hopf bifurcation and turning $P_{+}$into an unstable fixed point $P_{-}$. DNSP changes from a static state to a limit-cycle state, the onset of self-sustained oscillation [Fig. 2(c)]. The limit cycle touches the saddle point at another critical value $B=B_{2}$ of about $0.62 \mathrm{~T}$, undergoing a homoclinic bifurcation [Fig. 2(d)]. The corresponding period diverges when $B$ goes to $B_{2}$ because the phase velocity vanishes at the saddle point. These are exactly what were observed in the experiment: Below the onset field $B_{1}$, no self-sustained current oscillation could be observed because DNSP takes the steady state of $(1,0,0)$. For the field between $B_{1}$ and $B_{2}$, oscillating current appears because the only stable attractor is the limit cycle. The current period diverges as $B$ approaches $B_{2}$. The end of oscillating current was attributed to the homoclinic bifurcation at $B_{2}$.

In order to obtain the field dependence of the oscillation period, one needs to locate first the limit cycle for a given field. This can be done by using the Melnicov theory ${ }^{21,22}$ valid for a nearly conserved system of small $\alpha$ and $a$. It is well known that the energy dissipation rate 23

$$
f(U) \equiv \oint_{L(U)}\left(-\alpha \frac{d \vec{m}}{d t}-a \vec{m} \times \hat{x}\right) \cdot d \vec{m}
$$

is a function of equal energy contour $L(U)$, thus also a function of energy $U \equiv \frac{1}{2}\left(D_{x} m_{x}^{2}+D_{y} m_{y}^{2}+D_{z} m_{z}^{2}\right)-\vec{H} \cdot \vec{m} . f$ is the dissipated energy after the system moves along $L(U)$ once. Interestingly, the Melnicov functions of stable and unstable fixed points and limit cycles are zeros by definition. According to the Melnicov theory, the limit cycle is approximated by one particular $L\left(U^{*}\right)$ that satisfies $f\left(U^{*}\right)=0$. Figure $3($ a) is the $U$ dependence of $f(U)$ for $B=0.40,0.42$, and $0.62 \mathrm{~T}$ (from top to bottom) in the vicinity of $B_{0}, B_{1}$, and $B_{2}$, respectively. By definition, the energy contours for energy extremes $\left(P_{ \pm}\right.$ minima and maxima) are points, and the Melnicov function is zero there. These correspond to the far left and far right nodes in the figure. The origin (crossing of $x$ and $y$ axes) was chosen to be the energy of the saddle point. Zeros at which the $f$ curve have positive (negative) slopes correspond to stable (unstable) energy contour. To see this, consider a node $U^{*}$ with $\left.\frac{\partial f}{\partial U}\right|_{U^{*}}>0$ and a slight deviation of $U$ from $U^{*}$, say, $U>U^{*}$, $U$ should decrease after the system moving along the $L(U)$ once because $f(U)>f\left(U^{*}\right)=0$. Thus, the system tends to push $U$ back to $U^{*}$. For $B=0.40 \mathrm{~T}$, the zero slope of $f(U)$ at the node around $U=0.1$ corresponds to generation of a pair of limit cycles, leading to a semistable limit-cycle bifurcation $\left[f\left(U^{*}\right)=0,\left.\frac{\partial f}{\partial U}\right|_{U^{*}}=0\right]$. A slight increase of $B$ lowers the $f(U)$ curve near $U^{*}=0.1$ and the node splits into two. The left (right) one with negative (positive) slope corresponds to stable (unstable) limit cycle. The stable cycle $L_{+}$moves towards the SP, while the unstable cycle $L_{-}$moves towards $P_{+}$as $B$ increases. The $L_{-}$merges with $P_{+}$around $0.42 \mathrm{~T}$, and turns $P_{+}$into an unstable fixed point $P_{-}$(negative slope). This is a subcritical Hopf bifurcation. After further increase of $B$ to $0.62 \mathrm{~T}, L_{+}$touches saddle point SP and becomes a homoclinic loop, resulting in a homoclinic bifurcation. With the limit cycle for a given field in the window of $\left[B_{1}, B_{2}\right]$ located, the period of DNSP can be evaluated by

$$
\tau=\oint_{L\left(U^{*}\right)} \frac{d \vec{m}}{\frac{d \vec{m}}{d t}},
$$

where $d \vec{m} / d t$ is given by Eq. (1). As shown in Fig. 3(b), the period increases monotonically with field and diverges at $B_{2}$.

The electron in triplet state $\left(\left|T^{-}\right\rangle\right)$plays dual roles. It not only interacts with the nuclear spins by which spin flips generate DNSP, but also exerts a transversal torque on the DNSP. The self-sustained current oscillation comes from the periodic motion of DNSP along a limit cycle. The limit cycle originates from the instability of a static DNSP state under two competing forces: One is energy input from the tunneling electrons that drives the DNSP away from its static state, a fixed point. The other is the dissipation of the $\alpha$ term that tends to push the DNSP to its fixed point. ${ }^{24}$ The periodical motion of DNSP leads to the current oscillation. This theory explains why the current oscillation was only observed in the spin-blockaded regime and under a magnetic field. It also explains why the period can be several orders of magnitude longer than the typical spin precession period. ${ }^{25}$ In principle, the current oscillation reported here should exist 

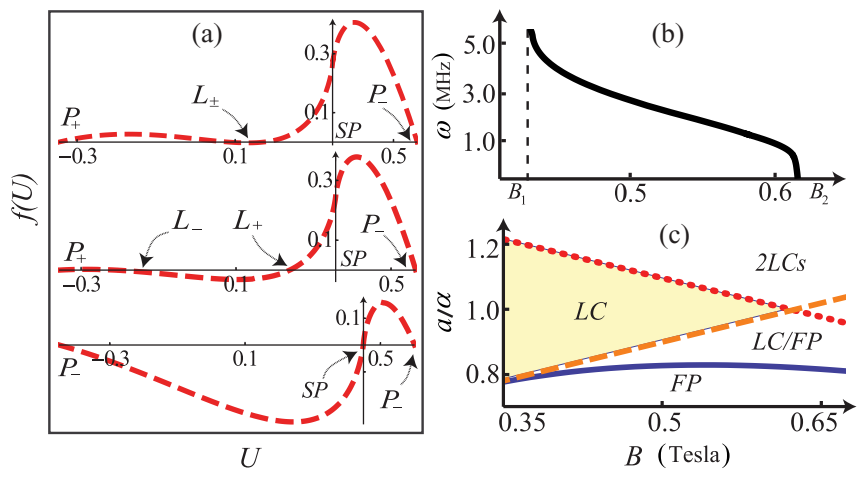

FIG. 3. (Color online) (a) The Melnicov curves $f(U)$ for $B=$ $0.40,0.42$, and $0.62 \mathrm{~T}$ (from top to bottom). Zeros with positive (negative) slopes are stable (unstable) attractors. The far left node corresponds to $P_{+}$and the far right node corresponds to $P_{-}$on the spheres of Fig. 2, while the origin (crossing of the two coordinate axes) was chosen to be at the value of the energy of SP. (b) Field dependence of self-sustained current oscillation frequency $\omega$. The oscillation occurs at $B_{1}$ and the period diverges at $B_{2}$. (c) Phase diagram in $B-a / \alpha$ plane. Dotted, dashed, and solid curves are for homoclinic, subcritical Hopf, and semistable limit-cycle bifurcations, respectively. FP, LC, LC/FP, and 2LCs denote one stable fixed point, one stable limit cycle, coexistence of stable limit cycle and fixed point, and two stable limit-cycle phases, respectively.

in systems with more than two dots in the spin-blockaded regime. However, it would be more likely to observe the current oscillation in a spin-blockaded double quantum dots given that it is easier to interact with fewer nuclear spins in a smaller space because there is no direct exchange coupling among nuclear spins, and nuclear spin alignment is mediated by polarized itinerant electron spins that suffer from the relaxation in a larger system. This is in a sharp contrast to the current oscillation observed in superlattices that originates from the negative differential resistance ${ }^{15}$ where it is only observed in superlattices with more than four wells. ${ }^{26}$ Although we believe that magnitude change of DNSP is irrelevant for the experimentally observed self-sustained current oscillation, a belief can not replace a scientific proof. Thus, it shall be interesting to see whether there are also field-induced Hopf and homoclinic bifurcations in Landau-Lifshitz-Bloch dynamics that govern spin dynamics, the magnitude of which can also vary.

Interestingly, if $a$ in Eq. (1) can vary independently from the field $B$, one can obtain the homoclinic bifurcation, subcritical Hopf bifurcation, and semistable limit-cycle bifurcation curves as a function of $B$ and $a / \alpha$ in a similar way as what we explained earlier. Our results are shown in Fig. 3(c). The phase diagram shows that Eq. (1) supports various stable phases (stable attractors), including single stable fixed point, coexistence of a stable fixed point and a stable limit cycle, and existence of two stable limit cycles. In summary, there are a number of predictions in our theory to be confirmed. According to our analysis, a limit-cycle solution can only exist when spin-transfer torque is large enough and self-sustained current oscillation appears and disappears when the tunneling current varies. Also, our theory predicts multiple stable attractors [Fig. 3(c)] in certain parameter regions, either the coexistence of limit cycle and fixed point or the existence of two limit cycles.

\section{CONCLUSIONS}

In conclusion, we showed that an external magnetic field can induce a Hopf bifurcation at a low field and a homoclinic bifurcation at a high field for DNSP in a spin-blockaded double quantum dot, between which is the magnetic field window for the self-sustained current oscillation. The amplitude and period of the oscillation become bigger and bigger as the field increases, in good agreement with the experimental findings. The period diverges at the homoclinic bifurcation in our model, which explains well why the period is of several orders of magnitude longer than the fundamental time scales.

\section{ACKNOWLEDGMENTS}

This work is supported by Hong Kong RGC Grants No. 604109 and No. RPC11SC05. X. R. Wang also acknowledges the hospitality of Kavli Institute for Theoretical Physics, Beijing, China.
*Corresponding author: phxwan@ust.hk

${ }^{1}$ M. A. Kastner, Phys. Today 46(1), 24 (1993).

${ }^{2}$ T. Brandes, Phys. Rep. 408, 315 (2005).

${ }^{3}$ Semiconductor Spintronics and Quantum Computation, edited by D. D. Awschalom, N. Samarth, and D. Loss (Springer, Berlin, 2002).

${ }^{4}$ T. Fujisawa, D. G. Austing, Y. Tokura, Y. Hirayama, and S. Tarucha, Nature (London) 419, 278 (2002); R. Hanson, B. Witkamp, L. M. K. Vandersypen, L. H. Willems van Beveren, J. M. Elzerman, and L. P. Kouwenhoven, Phys. Rev. Lett. 91, 196802 (2003).

${ }^{5}$ M. Dobers, K. Vonklitzing, and G. Weimann, Phys. Rev. B 38, 5453 (1988).

${ }^{6}$ K. Ono and S. Tarucha, Phys. Rev. Lett. 92, 256803 (2004).

${ }^{7}$ T. Inoshita and S. Tarucha, Phys. E (Amsterdam) 22, 422 (2004).

${ }^{8}$ C. Lopez-Monis, J. Inarrea, and G. Platero, New J. Phys. 13, 053010 (2011).
${ }^{9}$ M. S. Rudner and L. S. Levitov, Phys. Rev. Lett. 99, 036602 (2007).

${ }^{10}$ M. S. Rudner, F. H. L. Koppens, J. A. Folk, L. M. K. Vandersypen, and L. S. Levitov, Phys. Rev. B 84, 075339 (2011).

${ }^{11}$ J. Inarrea, G. Platero, and A. H. MacDonald, Phys. Rev. B 76, 085329 (2007).

${ }^{12}$ X. R. Wang and Q. Niu, Phys. Rev. B 59, R12755 (1999); X. R. Wang, J. N. Wang, B. Q. Sun, and D. S. Jiang, ibid. 61, 7261 (2000); Z. Z. Sun, H. T. He, J. N. Wang, S. D. Wang, and X. R. Wang, ibid. 69, 045315 (2004).

${ }^{13}$ M. Gullans, J. J. Krich, J. M. Taylor, H. Bluhm, B. I. Halperin, C. M. Marcus, M. Stopa, A. Yacoby, and M. D. Lukin, Phys. Rev. Lett. 104, 226807 (2010).

${ }^{14}$ S. Yin, Q. F. Sun, Z. Z. Sun, and X. R. Wang, J. Phys.: Condens. Matter 17, L183 (2005). 
${ }^{15}$ Z. Z. Sun and X. R. Wang, Phys. Rev. Lett. 97, 077205 (2006); X. R. Wang and Z. Z. Sun, ibid. 98, 077201 (2007).

${ }^{16}$ E. V. Charnaya, T. Loeser, D. Michel, C. Tien, D. Yaskov, and Y. A. Kumzerov, Phys. Rev. Lett. 88, 097602 (2002).

${ }^{17}$ J. Slonczewski, J. Magn. Magn. Mater. 159, L1 (1996).

${ }^{18}$ T. Fujisawa, T. H. Oosterkamp, W. G. van der Wiel, B. W. Broer, R. Aguado, S. Tarucha, and L. P. Kouwenhoven, Science 282, 932 (1998).

${ }^{19}$ J. R. Petta, A. C. Johnson, J. M. Taylor, E. A. Laird, A. Yacoby, M. D. Lukin, C. M. Marcus, M. P. Hanson, and A. C. Gossard, Science 309, 2180 (2005).

${ }^{20}$ J. Baugh, Y. Kitamura, K. Ono, and S. Tarucha, Phys. Rev. Lett. 99, 096804 (2007).
${ }^{21}$ S. H. Strogatz, Nonlinear Dynamics and Chaos (Westview, Cambridge, MA, 1994); L. Perko, Differential Equations and Dynamical Systems (Springer, New York, 2001).

${ }^{22}$ G. Bertotti, C. Serpico, I. D. Mayergoyz, A. Magni, M. d'Aquino, and R. Bonin, Phys. Rev. Lett. 94, 127206 (2005).

${ }^{23}$ Z. Z. Sun and X. R. Wang, Phys. Rev. B 73, 092416 (2006); 74, 132401 (2006).

${ }^{24}$ L. Landau and E. Lifshitz, Phys. Z. Sowjetunion 8, 153 (1935); T. L. Gilbert, Phys. Rev. 100, 1243 (1955).

${ }^{25}$ I. A. Merkulov, A. L. Efros, and M. Rosen, Phys. Rev. B 65, 205309 (2002).

${ }^{26}$ R. Escobedo, M. Carretero, L. L. Bonilla, and G. Platero, New J. Phys. 11, 013033 (2009). 